\title{
Nanoindentation testing as a powerful screening tool for assessing phase stability of nanocrystalline high-entropy alloys
}

\author{
Verena Maier-Kiener ${ }^{a}, B^{\text {Benjamin Schuh }}{ }^{b}$, Easo P. George ${ }^{c}$, Helmut Clemens $^{a}$, Anton Hohenwarter $^{b}$ \\ ${ }^{a}$ Department Physical Metallurgy and Materials Testing, Montanuniversität Leoben, 8700 Leoben, Austria \\ ${ }^{\mathrm{b}}$ Department Materials Physics, Montanuniversität Leoben, \& Austrian Academy of Sciences, Erich- \\ Schmid-Institute for Material Science, 8700 Leoben, Austria \\ 'Institute for Materials, Ruhr University Bochum, 44801 Bochum, Germany
}

\begin{abstract}
The equiatomic high-entropy alloy (HEA), CrMnFeCoNi, has recently been shown to be microstructurally unstable, resulting in a multi-phase microstructure after intermediate-temperature annealing treatments. The decomposition occurs rapidly in the nanocrystalline (NC) state and after longer annealing times in coarse-grained states. To characterize the mechanical properties of differently annealed NC states containing multiple phases, nanoindentation was used. The results revealed besides drastic changes in hardness, also for the first time significant changes in the Young's modulus and strain rate sensitivity. Nanoindentation of NC HEAs is, therefore, a useful complementary screening tool with high potential as a high throughput approach to detect phase decomposition, which can also be used to qualitatively predict the long-term stability of single-phase HEAs.
\end{abstract}

Keywords: Nanoindentation; high-entropy alloys; severe plastic deformation; high-pressure torsion; phase stability; nanocrystalline

(C) 2016. This manuscript version is made available under the Elsevier user license http://www.elsevier.com/open-access/userlicense/1.0/ 


\section{Introduction}

The Cantor alloy, an equiatomic CrMnFeCoNi alloy, is one of the few multi-principal-element alloys, or HEAs, that is a single-phase solid solution at room temperature after elevated temperature homogenization [1,2]. Since the first report of its existence in 2004 [3] the mechanical and physical properties has been thoroughly investigated[4-16] along with many other systems[17,18]. Recent studies have concentrated on phase stability as a function of temperature and revealed the formation of metallic and intermetallic phases at temperatures below $\sim 800{ }^{\circ} \mathrm{C}$. The first study [19] to demonstrate decomposition in this HEA dealt with its nanocrystalline (NC) state in which the decomposition occurred fast, within minutes. Subsequent reports dealt with larger grain sizes in the millimeter range (referred to as coarse-grained (CG) materials here) where the second phases were observed after much longer times $[20,21]$. The decomposition into a multiphase structure has the potential to significantly affect mechanical properties. For example, in the NC state, which has a relatively high strength even before annealing, the decomposition was accompanied by an increase of strength and decrease of ductility [19]. However, the impact on other important properties, such as Young's modulus or strain rate sensitivity (SRS), has not yet been investigated. The effects of decomposition on the mechanical properties of the CG HEA have not been reported, but it is a concern since grain boundary precipitation of deleterious intermetallic phases could potentially cause embrittlement.

For studying these decomposition effects mainly advanced microscopy techniques such as SEM (scanning electron microscopy) with BSE (backscattered electron) contrast, TEM (transmission electron microscopy) or even more localized three-dimensional APT (atom probe tomography) are used. Even though these methods are required to gain important insights into the complex microstructures, sample preparation, especially for TEM and APT, is time consuming. Moreover, the large compositional space of these new class of multi-principal element alloys also requires new, high throughput approaches, which can accelerate the discovery and development of alloy compositions with interesting and promising material properties [22-24] especially when a single-phase character for a certain application temperature is targeted. Therefore, a simple method would be beneficial that allows a faster and reliable screening and evaluation of possible decomposition effects in single-phase HE-alloys before advanced microstructural analysis methods are applied.

Here we evaluate the potential of nanoindentation testing as such a high-throughput approach with a case study on the NC-Cantor alloy. 
To this end, we characterize the mechanical behavior (hardness, Young's modulus and strain rate sensitivity, SRS) of the NC Cantor alloy after various annealing treatments and compare it to the behavior of CG materials. It will be demonstrated that the temperature dependent precipitation and redissolution of second phases can be monitored by changes in the Young's modulus of the NC-state, which can indirectly also provide qualitative information about the long-term stability of the HEAs having conventional grain sizes.

\section{Experimental}

The equiatomic HEA, CrMnFeCoNi, was synthesized by arc melting and drop-casting in pure $\mathrm{Ar}$ atmosphere followed by homogenization at $1200{ }^{\circ} \mathrm{C}$ for 48 hours as described elsewhere [19]. This state with grain size in the millimeter range will be referred to as cast and homogenized $(\mathrm{CH})$ material. Next, the HEA was processed by high-pressure torsion (HPT) using disks $8 \mathrm{~mm}$ in diameter and $0.8 \mathrm{~mm}$ thick. The pressure during deformation was $7.8 \mathrm{GPa}$, the rotational speed was 0.2 rotations/minute, and 5 rotations were applied. In addition, material with an approximate grain size of $6 \mu \mathrm{m}$ was analyzed (referred to as MG material here for its micrometer-sized grains), which had been fabricated by deformation processing and annealing as described elsewhere [9]. Grain sizes for the three different states are given in Table 1 . These material states were separately subjected to different isochronal anneals for $1 \mathrm{~h}$ each at temperatures from $200{ }^{\circ} \mathrm{C}$ to $1000^{\circ} \mathrm{C}$ and isothermal heat treatments at $450{ }^{\circ} \mathrm{C}$ up to $200 \mathrm{~h}$.

In order to investigate the mechanical properties of these heat treated conditions, nanoindentation experiments were performed, for which the specimens were ground and polished, first mechanically with SiC paper and then mechano-chemically with colloidal silica. Nanoindentation testing was carried out in the post-annealed conditions at room temperature using a platform nanoindenter G200 (KeysightTec, USA) equipped with a three-sided diamond Berkovich tip. Tip shape and frame stiffness calibrations were performed at regular intervals with fused silica following the Oliver \& Pharr method [25]. Depth dependent local mechanical properties were determined using a continuous stiffness measurement (CSM) unit, which measures the contact stiffness continuously by superimposing a sinusoidal load signal ( $45 \mathrm{~Hz}, 2 \mathrm{~nm}$ oscillation amplitude) on the normal indentation load. All nanoindentation experiments were performed in constant strain rate mode and at thermal drift rates of less than $0.1 \mathrm{~nm} / \mathrm{s}$. Hardness and Young's modulus (assuming a Poisson's ratio for the HEA of 0.25 [7]) were determined by constant strain rate indentation $\left(0.05 \mathrm{~s}^{-1}\right)$ to a preset indentation depth of $2500 \mathrm{~nm}$. The calculated depth 
dependent mechanical properties were further averaged between $1000 \mathrm{~nm}$ and $1900 \mathrm{~nm}$ indentation depth. Local SRS data were calculated from nanoindentation strain rate jump tests [26] during which the applied strain rate was changed abruptly every $500 \mathrm{~nm}$, starting at $0.05 \mathrm{~s}^{-1}$, then dropping to 0.005 , followed by an increase to 0.05 , then dropping to $0.001 \mathrm{~s}^{-1}$, and so on. Thermally activated processes were quantified as described elsewhere $[27,28]$ by calculating the SRS $m(m=\partial \ln H / \partial \ln \dot{\varepsilon})$ and activation volume $V^{*}\left(V^{*}=C^{*} \cdot \sqrt{3} \cdot k_{B} \cdot T / m H\right)$ from the measured dependence of hardness $H$ on strain rate $\dot{\varepsilon}$ (with $C^{*}$ : constraint factor of $2.8, k_{b}$ : Boltzmann constant, $T$ : room-temperature $25^{\circ} \mathrm{C}$ ).

\section{Results \& Discussion}

\section{Microstructure}

The microstructural developments and the chemical decomposition of the material during annealing has been analyzed in detail (TEM, APT) and discussed in a previous paper published by Schuh et al. [19]. A short summary of the most important microstructural features is presented in Fig. 1: By applying HPT on the single-phase solid solution HEA, a NC microstructure with grain size of less than $50 \mathrm{~nm}$ can be achieved, as shown in the TEM micrograph in Figure 1a. In this state the single-phase solid solution character is retained, which was confirmed by APT measurements. Further annealing treatments of the NC materials led to a decomposition of the originally single-phase alloy into a state with more than one phase, including some intermetallic phases. These precipitates and new phases can be detected by advanced microscopy techniques such as TEM ([19]), by APT to probe very small volumes, see Figure 1b and by SEM (Figure 1c-e). Evidence of a newly formed crystal structure is also indicated by additional diffraction rings in TEM diffraction mode [19]. For further details and discussion the reader is referred to an earlier publication by some of the present authors (Schuh et al.[19]).

\section{Mechanical behavior - Hardness \& Young's Modulus}

After HPT, the as-deformed NC HEA has an average hardness of $6.5 \pm 0.04 \mathrm{GPa}$ at room temperature, which is significantly higher than the room-temperature hardness, $2.5 \pm 0.04 \mathrm{GPa}$, of HEA specimens that were not subjected to HPT (CH and MG states) (Figure 2). Representative load-displacement curves, hardness and Young's modulus as well as residual impressions for NC and CH HEA are shown in Figure 2 
(a: mechanical data and b. residual impressions). For both microstructural states, there is similar independence of Young's modulus on indentation depth. However, the applied load and thus the hardness is strongly influenced by the grain size, with the NC HEA showing a depth independent hardness, while the $\mathrm{CH}$ state is significantly influenced by indentation size effect (ISE). Focusing more on the average values and starting with the NC as-deformed condition, the hardness of the NC HEA increases further after isochronal and isothermal annealing treatments (Fig. 3a and Fig. 4, respectively). For $1 \mathrm{~h}$ anneals (Fig. 3a) hardness increases with annealing temperature and reaches a maximum of $7.64 \pm 0.06 \mathrm{GPa}$ at $500{ }^{\circ} \mathrm{C}$. Afterwards, hardness decreases and falls to $\sim 2.2 \mathrm{GPa}$ for temperatures of $800^{\circ} \mathrm{C}$ and higher. Heat treatments were also performed on the MG (green circles) and $\mathrm{CH}$ material (red triangles), both of which exhibited no hardness variation as a function of annealing temperature (Fig. 3a). The MG sample with an approximate grain size of $6 \mu \mathrm{m}$ shows slightly higher hardness values than the $\mathrm{CH}$ material, which is likely related to the larger grain size of the latter (order of millimeters in comparison to several $\mu \mathrm{m}$ ). For the temperature at which maximum hardness was measured after 1hour heat treatments, additional isothermal heat treatments were conducted. The results of isothermal heat treatments at $450{ }^{\circ} \mathrm{C}$, for times of 5 minutes to $200 \mathrm{~h}$, are shown in Fig. 4 . There is a significant hardness increase with annealing time, reaching a maximum of around $11 \mathrm{GPa}$ after $100 \mathrm{~h}$, which shows that equilibrium is not reached after $1 \mathrm{~h}$ at $450{ }^{\circ} \mathrm{C}$ (Fig. 3a). Beyond this maximum there is a slight decrease to $10 \mathrm{GPa}$, possibly due to grain growth. The standard deviations for these heat treatment conditions are relatively small as indicated by the small error bars. The present hardness trends agree well with the macroscopic Vickers hardness measurements performed previously by Schuh et al. [19] where the changes were correlated with the formation of metallic and intermetallic nanophases by advanced characterization techniques (TEM and APT). For short annealing times, a secondary contribution from dislocation annihilation was suggested, which is a hardening mechanism reported in NC metals [29,30].

In addition to the hardness, nanoindentation testing can be used to determine Young's modulus from the contact stiffness. The CH and MG states have Young's moduli between 205 and $220 \mathrm{GPa}$, depending mainly on the grain orientation, whereas for the initial NC state after HPT a Young's modulus of $205 \pm 0.5 \mathrm{GPa}$ was determined, Fig. 3b, and Fig. 4. These values for the CG, non-deformed condition are comparable to those determined for this HEA in the MG state by resonant ultrasonic spectroscopy (202 GPa,[8]). Upon annealing, the modulus of the NC material first increases to a maximum of $252 \mathrm{GPa}$ at $550{ }^{\circ} \mathrm{C}$, then remains fairly constant up to $700{ }^{\circ} \mathrm{C}$, beyond which it decreases significantly at $800{ }^{\circ} \mathrm{C}$ and higher temperatures (Fig. 3b). Moreover, there is very pronounced scatter in the modulus within these higher-temperature annealed states as indicated by the significant error bars. Similar scatter is also 
present in the Young's modulus obtained from all experiments performed on the MG and CH materials regardless of annealing temperature. Within the limits of this scatter, their moduli remain roughly constant as a function of annealing temperature. After the isothermal treatments at $450{ }^{\circ} \mathrm{C}$ (Fig. 4 ), the Young's modulus of the NC material increases slightly, to $262 \mathrm{GPa}$ after $200 \mathrm{~h}$, which indicates again the lack of equilibrium after 1 hour.

For all the above results, a significant effect of pile-up, which can influence the hardness and modulus determined using the Oliver-Pharr method [25], can be ruled out since our indentations look microscopically similar after the different heat treatments. Fig. $2 \mathrm{~b}$ shows representative light microscopy images of indents in the $\mathrm{CH}$ and NC HEAs along with their mechanical data. The impressions in the $\mathrm{CH}$ material are affected by crystallographic orientation, showing some orientation dependent irregular pileup formation around each indentation. In contrast, the NC HEA shows homogenous pile-up formation, but with smaller impressions overall since the maximum indentation load was reached at around $2200 \mathrm{~nm}$ before the pre-set indentation depth of $2500 \mathrm{~nm}$ was reached.

As mentioned before, the hardness increase after annealing is mainly due to precipitation hardening plus a smaller contribution from dislocation annihilation processes in the early stages. At present, these two mechanisms cannot be fully separated, however, for the hardening by annealing, which is correlated to a change in the defect structure, no change in the Young's modulus is expected. Thus, the detected changes in Young's modulus can be directly related to the formation of additional phases during annealing, since the newly formed metallic and intermetallic phases can be assumed to have higher moduli than the single-phase HEA, especially the intermetallic phases. These phases, namely, a MnNi phase, a Cr-rich phase, and a Fe-Co phase have been found in previous studies and their formation kinetics depend markedly on annealing time and temperature $[19,21])$ By the same token, the maximum in the modulus plot persists to higher temperatures than the maximum in hardness (Fig. 3a,b). This is because hardness is affected by three factors: the precipitation of additional nanophases, their stability as well as their average grain size. The hardness measurements on the heat-treated structure suggest that the grain size of the fcc HEA matrix increases for annealing temperatures higher than $600{ }^{\circ} \mathrm{C}$ (Fig. 3)) and for lower annealing temperatures exposed to longer times (Fig. 4: $t>100 \mathrm{~h}$ ). The dissolution of the precipitates however is not directly linked to the hardness decrease as can be seen from the SEM images (Figure 1c-e). In contrast, the modulus is unaffected by grain size and therefore remains also high to higher temperatures even though hardness decreases. This implies that as long as the modulus remains high, it is a qualitative indication that additional phases are present, which is also consistent with our earlier microstructural investigations ([19], Figure1c-e). At annealing temperatures of $800{ }^{\circ} \mathrm{C}$ and higher, the modulus drops significantly and converges to those of the $\mathrm{CH}$ and MG HEAs. This can be 
directly correlated to a complete dissolution of the nanophases and restoration of the single phase, as shown by our previous microstructural analyses ([19] and Figure 1e). However, there is grain coarsening at elevated temperatures (see also Figure 1c-e), which leads to more pronounced scatter in the data as the tip size becomes small relative to the grain size resulting in different grain orientations being probed. A clear proof for the interrelation between phase formation in the NC state and the changes in Young's modulus can be seen in the mechanical behavior of the non-HPT samples. The different annealing treatments lead to no significant changes in the modulus (similar to what was described earlier for hardness). This is consistent with nanophase precipitation occurring only in the NC HEA and not in the MG and $\mathrm{CH}$ HEAs for the present set of experimental conditions and the investigated time span. Although the mechanical consequences of nanophase formation in the NC HEA can be detected even after low annealing temperatures, the continued evolution of hardness and modulus with time show that equilibrium is not reached after $1 \mathrm{~h}$ anneals at $450^{\circ} \mathrm{C}$ (Figures $1 \mathrm{~b}$ and 4). Nevertheless, these properties serve as useful precursor indicators of phase instabilities thereby avoiding the need for tedious microstructural analyses.

The future development of new alloys in the vast compositional space of HEAs requires experimental methods where both mechanical properties and such decomposition tendencies can be efficiently probed in a timely manner, as also Miracle at al. [22] have recently pointed out. For this purpose, the NC state has the following advantage; when the NC state is synthesized by severe plastic deformation, e.g., HPT, the alloy composition is identical to that of materials with coarser grains. However, its decomposition kinetics are significantly faster [19-21], which saves time. Furthermore, in the NC state, the larger indenter tip size compared to the microstructure results in a more representative volume being probed. By rapidly probing the phase stability of such NC HEAs it is possible to obtain results that are also relevant for coarser grained materials. As a further step and refinement of the present study the mechanical investigation of the individual newly formed phases could be envisaged. This would help to obtain a direct, quantitative correlation between measured indentation Young's modulus and the (intermetallic) phase fraction, which is however cumbersome due to the multiphase character of the alloy and beyond the scope of this paper. What we have shown here is that nanoindentation can be a powerful qualitative tool to probe decomposition tendencies as a first step, following which detailed microstructure characterization can be performed on a limited number of samples in the composition and processing conditions of interest.

\section{Thermal activation}


Besides determining the hardness and Young's modulus (as shown in Fig. 3), nanoindentation can also provide basic information on thermally activated deformation processes through characterization of SRS. Fig. 5 shows nanoindentation strain-rate jump results for the three material states considered in this study, i.e. MG, $\mathrm{CH}$ and NC. All the load-displacement curves are clearly affected by the strain rate changes showing some transient behavior after each abrupt strain rate change, Fig. 5a. The corresponding Young's modulus (inset in Fig. 5a), however, is not affected by the strain rate changes, exhibiting only increased scatter at lower strain rates due to the longer indentation time and thus more data points [31]. In contrast, the hardness is strongly affected by the strain rate changes, Fig. 5b. While for the $\mathrm{CH}$ and MG materials an indentation size effect (ISE) is found in combination with some SRS, the NC state shows pronounced SRS paired with a depth independent hardness (i.e., no ISE). Moreover, the hardness changes in the NC material occur abruptly without any pronounced transient behavior. This can be seen more clearly in Fig. 5c, which is a magnified view of one of the strain rate changes (from $0.05 \mathrm{~s}^{-1}$ to $0.001 \mathrm{~s}^{-1}$ ). While the $\mathrm{CH}$ and MG data are dominated by an ISE in combination with a pronounced transient behavior, the NC state shows some interesting yield point phenomena. After each strain-rate jump to a lower strain rate, the hardness first drops without any pronounced transient behavior, followed by a further, unexpected small increase to an intermediate hardness maximum. Beyond that point, the hardness again decreases slightly. This indicates that the strain rate dependent hardness changes are also dependent on the grain size. Moreover, the resultant hardness changes show distinct differences among the different material states. The largest hardness change, $\Delta \mathrm{H}$, of $0.3 \mathrm{GPa}$ occurs in the NC state, followed by the MG state $(\sim 0.2 \mathrm{GPa})$, with the $\mathrm{CH}$ condition having the smallest $\Delta \mathrm{H}$ $(0.1 \mathrm{GPa})$. To quantify this relationship between hardness difference and strain rate changes, the SRS $m$ was calculated. The value of $m$ increases as the grain size decreases, whereas the corresponding activation volumes show the opposite trend, see Table 1.

The nanoindentation strain rate jump experiments show that thermally activated processes are additionally influencing the overall deformation behavior of these materials. By applying simple nanoindentation methods, it was possible to probe time dependent mechanical properties of three different microstructural states with limited amount of sample volume. As expected for an NC fcc material, the higher SRS of the NC state can be explained by dislocation interactions with the grain boundaries, which has also been found in many other nanostructured fcc materials [32]. For example, the hardness change without a pronounced transient behavior paired with a distinct yield point phenomena in the NC condition was previously also reported for NC NiFe alloys in micron sized tension experiments [33]. At present, the phenomenon is still not fully understood, however it is not found in 
pure NC Ni [31], suggesting that it may be related to high alloying contents. Another interesting feature is that, the $\mathrm{CH}$ and MG states also show some pronounced hardness changes after the strain rate jumps, which is not usually observed in pure coarse-grained or single crystalline fcc metals [26-28,32]. Generally this behavior resembles that seen in single crystal and coarse-grained bcc metals and was only once before reported for this material class [34]. In bcc crystal structure the deformation is governed by thermal activation over the Peierls stress, which leads to a pronounced SRS below a critical, material specific temperature $T_{c}$. Since both coarse grained conditions, $\mathrm{CH}$ and MG, show SRS values around 0.01 (0.008 (CH), $0.014(\mathrm{MG}))$ paired with a relatively small activation volume $V^{*}$ for fcc metals $\left(69 \cdot b^{3}(\mathrm{CH})\right.$, $\left.42 \cdot b^{3}(M G)\right)$, these first local results suggest a high lattice friction stress that has to be overcome by thermal activation.

By decreasing the grain size from $\mathrm{CH}$ to $\mathrm{MG}$ to $\mathrm{NC}$ the measured hardness differences become larger and the shape of the transient significantly changes. This might be an additional indication of an interplay between lattice resistance and thermally activated dislocation interactions with the grain boundaries.

\section{Conclusions}

The CrMnFeCoNi HEA was synthesized by high-pressure torsion as a single-phase, nanocrystalline material having the fcc crystal structure. The alloy was then subjected to various isochronal and isothermal heat treatments to decompose it into multiple metallic and intermetallic phases, as proven by advanced high-resolution microscopy techniques. Nanoindentation testing was successfully used to measure various room-temperature mechanical properties of these material states, and their properties were compared to those of coarser grained materials. The results can be summarized as follows:

(i) Hardness and Young's modulus exhibit a similar tendency upon annealing, showing an initial increase with annealing temperature followed by a decrease at higher temperatures.

(ii) The initial hardness increase is mainly due to the newly formed nanophases while the subsequent softening is due to grain growth and dissolution of the second phases. In contrast, the Young's modulus is not affected by grain size and is mainly dependent on the presence (or absence) of secondary phases. Therefore, nanoindentation of NC microstructures is a direct and fast way to probe the phase stability especially of single-phase HEAs and can be used as a complementary high-throughput screening approach to probe many different chemical compositions in short times. It also correlates with the longterm behavior of coarser grained materials and can therefore be used for screening purposes.

(iii) High strain rate sensitivity was found for all tested conditions, irrespective of the grain size. 
Finally, some questions still remain, e.g. for the CG states an increased fluctuation of Young's modulus suggests strong elastic anisotropy, which needs to be characterized. The high strain rate sensitivity of the coarse grained HEA is not typical of other fcc materials and the responsible mechanisms therefore need to be further studied.

\section{Acknowledgements}

The authors acknowledge funding by the European Research Council under grant number 340185 (V.M.K.) as well as by the Austrian Science Fund (FWF) in the framework of Research Project P26729-N19 (B.S. \& A.H.). Further financial support by the Austrian Federal Government (837900) within the framework of the COMET Funding Programme is highly appreciated. The HEA was produced while E.P.G. was at the Oak Ridge National Laboratory sponsored by the U.S. Department of Energy, Office of Science, Basic Energy Sciences, Materials Sciences and Engineering Division; E.P.G. also acknowledges DFG funding in Germany through project GE 2736/1-1.

\section{References:}

[1] F. Otto, Y. Yang, H. Bei, E.P. George, Relative effects of enthalpy and entropy on the phase stability of equiatomic high-entropy alloys, Acta Mater. 61 (2013) 2628-2638. doi:10.1016/j.actamat.2013.01.042.

[2] M. Laurent-Brocq, A. Akhatova, L. Perrière, S. Chebini, X. Sauvage, E. Leroy, Y. Champion, Insights into the phase diagram of the CrMnFeCoNi high entropy alloy, Acta Mater. 88 (2015) 355-365. doi:10.1016/j.actamat.2015.01.068.

[3] B. Cantor, I.T.H. Chang, P. Knight, A.J.B. Vincent, Microstructural development in equiatomic multicomponent alloys, Mater. Sci. Eng. A. 375-377 (2004) 213-218. doi:10.1016/j.msea.2003.10.257.

[4] A. Gali, E.P. George, Tensile properties of high- and medium-entropy alloys, Intermetallics. 39 (2013) 74-78. doi:10.1016/j.intermet.2013.03.018.

[5] N. Stepanov, M. Tikhonovsky, N. Yurchenko, D. Zyabkin, M. Klimova, S. Zherebtsov, A. Efimov, G. Salishchev, Effect of cryo-deformation on structure and properties of CoCrFeNiMn high-entropy alloy, Intermetallics. 59 (2015) 8-17. doi:10.1016/j.intermet.2014.12.004.

[6] A.J. Zaddach, C. Niu, C.C. Koch, D.L. Irving, Mechanical Properties and Stacking Fault Energies of NiFeCrCoMn High-Entropy Alloy, JOM. 65 (2013) 1780-1789. doi:10.1007/s11837-013-0771-4.

[7] G. Laplanche, P. Gadaud, O. Horst, F. Otto, G. Eggeler, E.P. George, Temperature dependencies of the elastic moduli and thermal expansion coefficient of an equiatomic, single-phase $\mathrm{CoCrFeMnNi}$ 
high-entropy alloy, J. Alloys Compd. 623 (2015) 348-353. doi:10.1016/j.jallcom.2014.11.061.

[8] A. Haglund, M. Koehler, D. Catoor, E.P. George, V. Keppens, Polycrystalline elastic moduli of a high-entropy alloy at cryogenic temperatures, Intermetallics. 58 (2015) 62-64. doi:10.1016/j.intermet.2014.11.005.

[9] B. Gludovatz, A. Hohenwarter, D. Catoor, E.H. Chang, E.P. George, R.O. Ritchie, A fractureresistant high-entropy alloy for cryogenic applications, Science. 345 (2014) 1153-1158. doi:10.1126/science.1254581.

[10] D.-H. Lee, I.-C. Choi, M.-Y. Seok, J. He, Z. Lu, J.-Y. Suh, M. Kawasaki, T.G. Langdon, J. Jang, Nanomechanical behavior and structural stability of a nanocrystalline CoCrFeNiMn high-entropy alloy processed by high-pressure torsion, J. Mater. Res. 30 (2015) 2804-2815. doi:10.1557/jmr.2015.239.

[11] K.-Y. Tsai, M.-H. Tsai, J.-W. Yeh, Sluggish diffusion in Co-Cr-Fe-Mn-Ni high-entropy alloys, Acta Mater. 61 (2013) 4887-4897. doi:10.1016/j.actamat.2013.04.058.

[12] J.Y. He, C. Zhu, D.Q. Zhou, W.H. Liu, T.G. Nieh, Z.P. Lu, Steady state flow of the FeCoNiCrMn high entropy alloy at elevated temperatures, Intermetallics. 55 (2014) 9-14. doi:10.1016/j.intermet.2014.06.015.

[13] W. Woo, E.-W. Huang, J.-W. Yeh, H. Choo, C. Lee, S.-Y. Tu, In-situ neutron diffraction studies on high-temperature deformation behavior in a CoCrFeMnNi high entropy alloy, Intermetallics. 62 (2015) 1-6. doi:10.1016/j.intermet.2015.02.020.

[14] G.D. Sathiaraj, P.P. Bhattacharjee, Effect of starting grain size on the evolution of microstructure and texture during thermo-mechanical processing of CoCrFeMnNi high entropy alloy, J. Alloys Compd. 647 (2015) 82-96. doi:10.1016/j.jallcom.2015.06.009.

[15] S. Huang, W. Li, S. Lu, F. Tian, J. Shen, E. Holmström, L. Vitos, Temperature dependent stacking fault energy of FeCrCoNiMn high entropy alloy, Scr. Mater. 108 (2015) 44-47. doi:10.1016/j.scriptamat.2015.05.041.

[16] T.M. Smith, M.S. Hooshmand, B.D. Esser, F. Otto, D.W. McComb, E.P. George, M. Ghazisaeidi, M.J. Mills, Atomic-scale characterization and modeling of $60^{\circ}$ dislocations in a high-entropy alloy, Acta Mater. 110 (2016) 352-363. doi:10.1016/j.actamat.2016.03.045.

[17] Y. Zhang, T.T. Zuo, Z. Tang, M.C. Gao, K.A. Dahmen, P.K. Liaw, Z.P. Lu, Microstructures and properties of high-entropy alloys, Prog. Mater. Sci. 61 (2014) 1-93. doi:10.1016/j.pmatsci.2013.10.001.

[18] E.J. Pickering, N.G. Jones, High-entropy alloys: a critical assessment of their founding principles and future prospects, Int. Mater. Rev. 6608 (2016) 1-20. doi:10.1080/09506608.2016.1180020. 
[19] B. Schuh, F. Mendez-Martin, B. Völker, E.P. George, H. Clemens, R. Pippan, A. Hohenwarter, Mechanical properties, microstructure and thermal stability of a nanocrystalline CoCrFeMnNi high-entropy alloy after severe plastic deformation, Acta Mater. 96 (2015) 258-268. doi:10.1016/j.actamat.2015.06.025.

[20] E.J. Pickering, R. Muñoz-Moreno, H.J. Stone, N.G. Jones, Precipitation in the equiatomic highentropy alloy CrMnFeCoNi, Scr. Mater. 113 (2016) 106-109. doi:10.1016/j.scriptamat.2015.10.025.

[21] F. Otto, A. Dlouhý, K.G. Pradeep, M. Kuběnová, D. Raabe, G. Eggeler, E.P. George, Decomposition of the single-phase high-entropy alloy $\mathrm{CrMnFeCoNi}$ after prolonged anneals at intermediate temperatures, Acta Mater. 112 (2016) 40-52. doi:10.1016/j.actamat.2016.04.005.

[22] D. Miracle, B. Majumdar, K. Wertz, S. Gorsse, New strategies and tests to accelerate discovery and development of multi-principal element structural alloys, Scr. Mater. (2016) 6-11. doi:10.1016/j.scriptamat.2016.08.001.

[23] K.G. Pradeep, C.C. Tasan, M.J. Yao, Y. Deng, H. Springer, D. Raabe, Non-equiatomic high entropy alloys: Approach towards rapid alloy screening and property-oriented design, Mater. Sci. Eng. A. 648 (2015) 183-192. doi:10.1016/j.msea.2015.09.010.

[24] A. Marshal, K.G. Pradeep, D. Music, S. Zaefferer, P.S. De, J.M. Schneider, Combinatorial synthesis of high entropy alloys: Introduction of a novel, single phase, body-centered-cubic FeMnCoCrAl solid solution, J. Alloys Compd. 691 (2017) 683-689. doi:10.1016/j.jallcom.2016.08.326.

[25] W.C. Oliver, G.M. Pharr, An improved technique for determining hardness and elastic modulus using load and displacement sensing indentation experiments, J. Mater. Res. 7 (1992) 1564-1583. doi:10.1557/JMR.1992.1564.

[26] V. Maier, C. Schunk, M. Göken, K. Durst, Microstructure-dependent deformation behaviour of bcc-metals - indentation size effect and strain rate sensitivity, Philos. Mag. 95 (2014) 1766-1779. doi:10.1080/14786435.2014.982741.

[27] Q. Wei, S. Cheng, K.T. Ramesh, E. Ma, Effect of nanocrystalline and ultrafine grain sizes on the strain rate sensitivity and activation volume: fcc versus bcc metals, Mater. Sci. Eng. A. 381 (2004) 71-79.

[28] K. Durst, V. Maier, Dynamic nanoindentation testing for studying thermally activated processes from single to nanocrystalline metals, Curr. Opin. Solid State Mater. Sci. 19 (2015) 340-353. doi:10.1016/j.cossms.2015.02.001.

[29] O. Renk, a. Hohenwarter, K. Eder, K.S. Kormout, J.M. Cairney, R. Pippan, Increasing the strength of nanocrystalline steels by annealing: Is segregation necessary?, Scr. Mater. 95 (2015) 27-30. 
doi:10.1016/j.scriptamat.2014.09.023.

[30] X. Huang, N. Hansen, N. Tsuji, Hardening by Annealing and Softening by Deformation in Nanostructured Metals, Science. 312 (2006) 249-251. doi:10.1126/science.1124268.

[31] V. Maier, K. Durst, J. Mueller, B. Backes, H.W. Höppel, M. Göken, Nanoindentation strain-rate jump tests for determining the local strain-rate sensitivity in nanocrystalline $\mathrm{Ni}$ and ultrafinegrained Al, J. Mater. Res. 26 (2011) 1421-1430. doi:10.1557/jmr.2011.156.

[32] H.W. Höppel, J. May, P. Eisenlohr, M. Göken, Strain-rate sensitivity of ultrafine-grained materials, Zeitschrift Für Met. 96 (2005) 566-571. doi:10.3139/146.101071.

[33] S. Van Petegem, J. Zimmermann, H. Van Swygenhoven, Yield point phenomenon during strain rate change in nanocrystalline Ni-Fe, Scr. Mater. 65 (2011) 217-220. doi:10.1016/j.scriptamat.2011.04.007.

[34] M. Komarasamy, N. Kumar, R.S. Mishra, P.K. Liaw, Anomalies in the deformation mechanism and kinetics of coarse-grained high entropy alloy, Mater. Sci. Eng. A. 654 (2016) 256-263. doi:10.1016/j.msea.2015.12.063. 
Figure 1. Advanced microscopy results of HEA after deformation by HPT and annealing, a) TEM image of as deformed, NC condition, b) APT reconstructions of 3 annealed HPT conditions at $450^{\circ} \mathrm{C}$ for $5 \mathrm{~min}, 1 \mathrm{~h}$ and $15 \mathrm{~h}$. and c) -e) microstructure by SEM (BSE contrast) of HPT specimens after $1 \mathrm{~h}$ heat treatments at 700,750 and $800^{\circ} \mathrm{C}$, respectively (intermetallic phases are indicated by circles $\mathrm{c}$ and d). This figure is an adapted reproduction from Schuh et al. [19], which is an open access article under the CC BY license (http://creativecommons.org/licenses/by/4.0/)

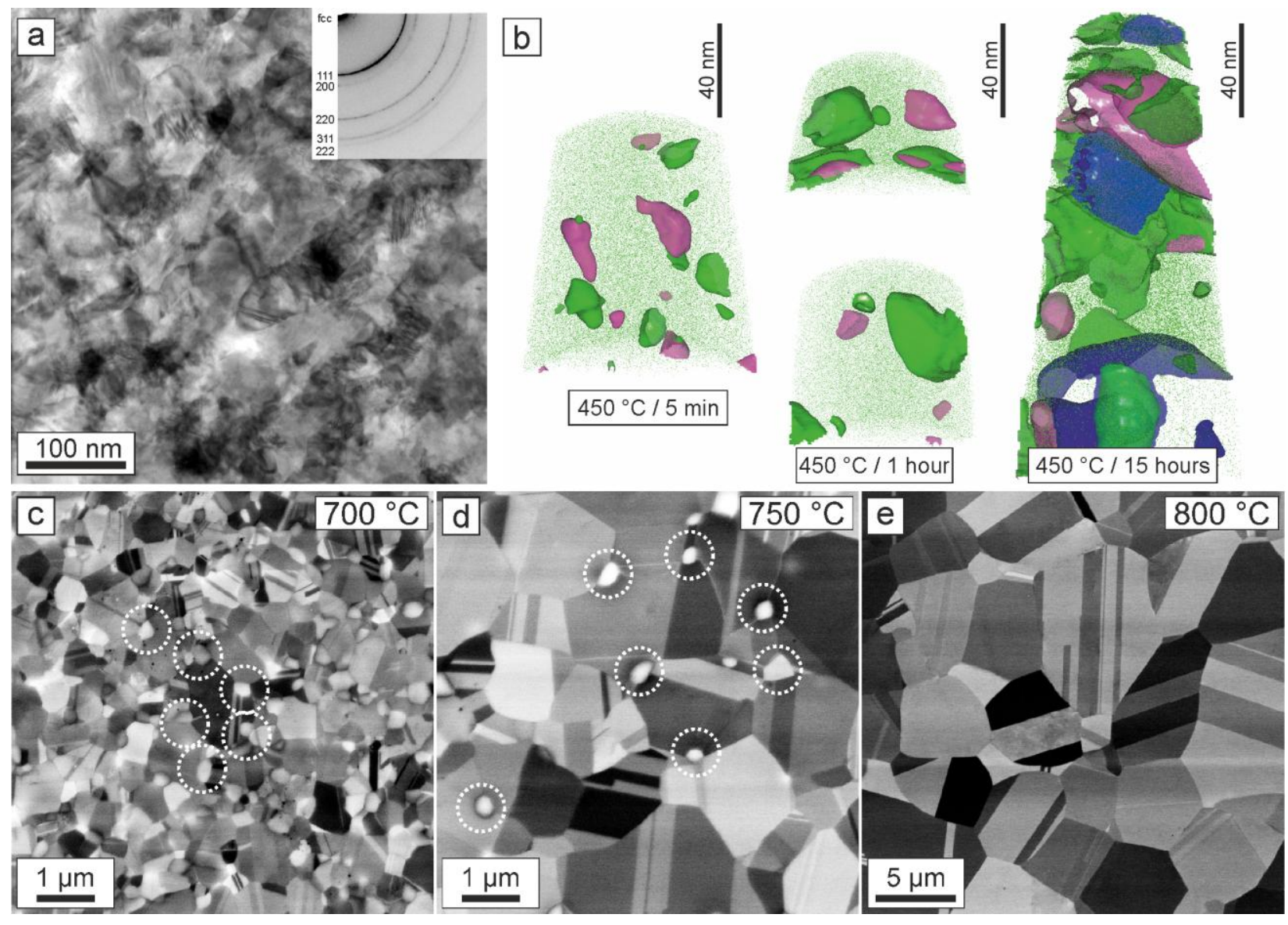


Figure 2. Nanoindentation results a) mechanical data (Young's modulus, hardness and load vs displacement) for $\mathrm{CH}$ and $\mathrm{NC}$ materials after $1 \mathrm{~h}$ anneal at $300^{\circ} \mathrm{C}$ and b) corresponding light microscopy images of residual indentation arrays (circles indicate the indents whose indentation data are displayed).
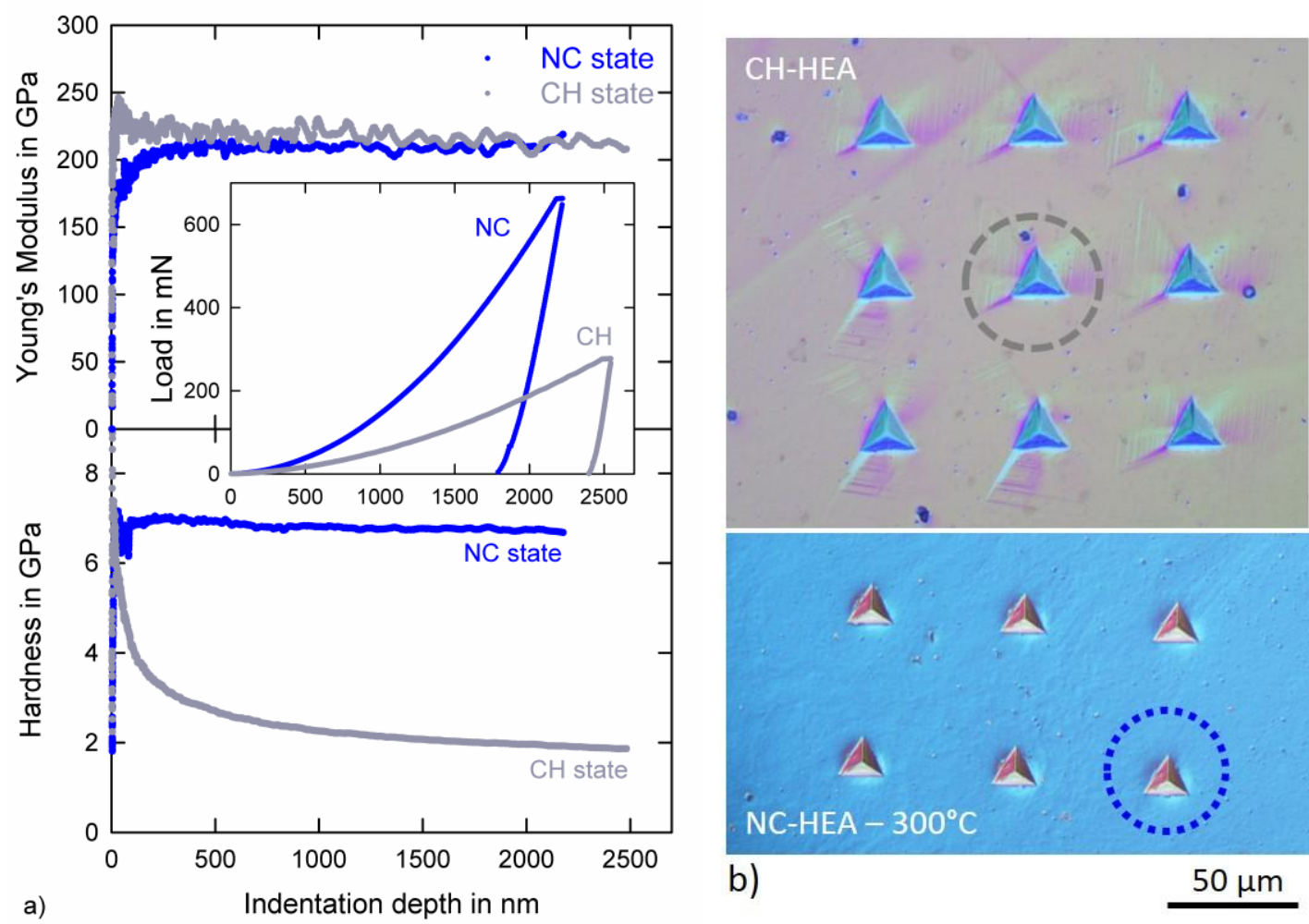
Figure 3. Nanoindentation results for NC, MG, and $\mathrm{CH}$ materials. a) Hardness and b) Young's modulus measured at room temperature after $1 \mathrm{~h}$ isochronal heat treatments at different annealing temperatures. (For interpretation of the references to color in this figure legend, the reader is referred to the web version of this article.)

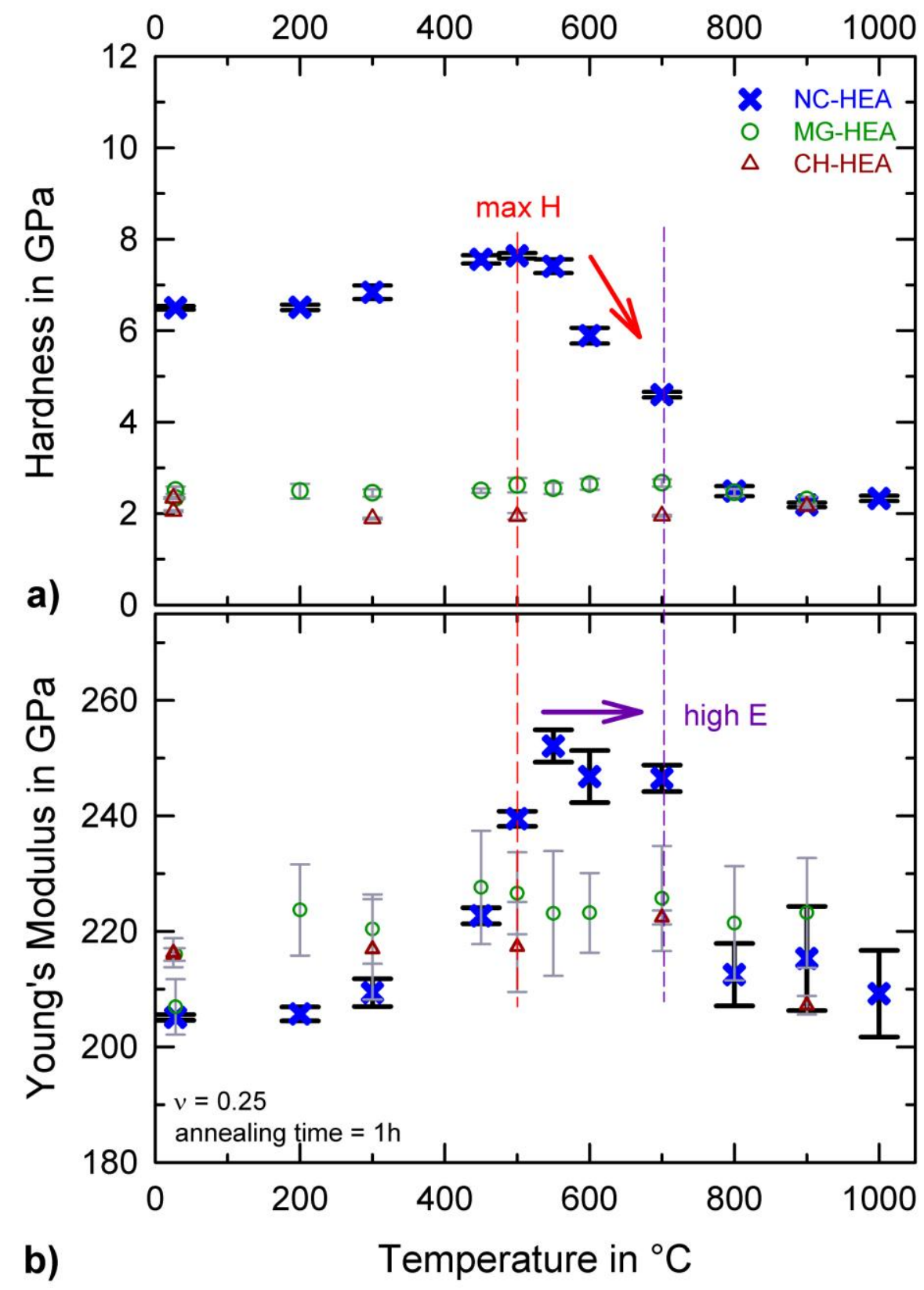


Figure 4. Nanoindentation results for $\mathrm{NC}, \mathrm{MG}$, and $\mathrm{CH}$ materials. Room-temperature hardness and Young's modulus after isothermal heat treatments at $450{ }^{\circ} \mathrm{C}$ for various times. (For interpretation of the references to color in this figure legend, the reader is referred to the web version of this article.)

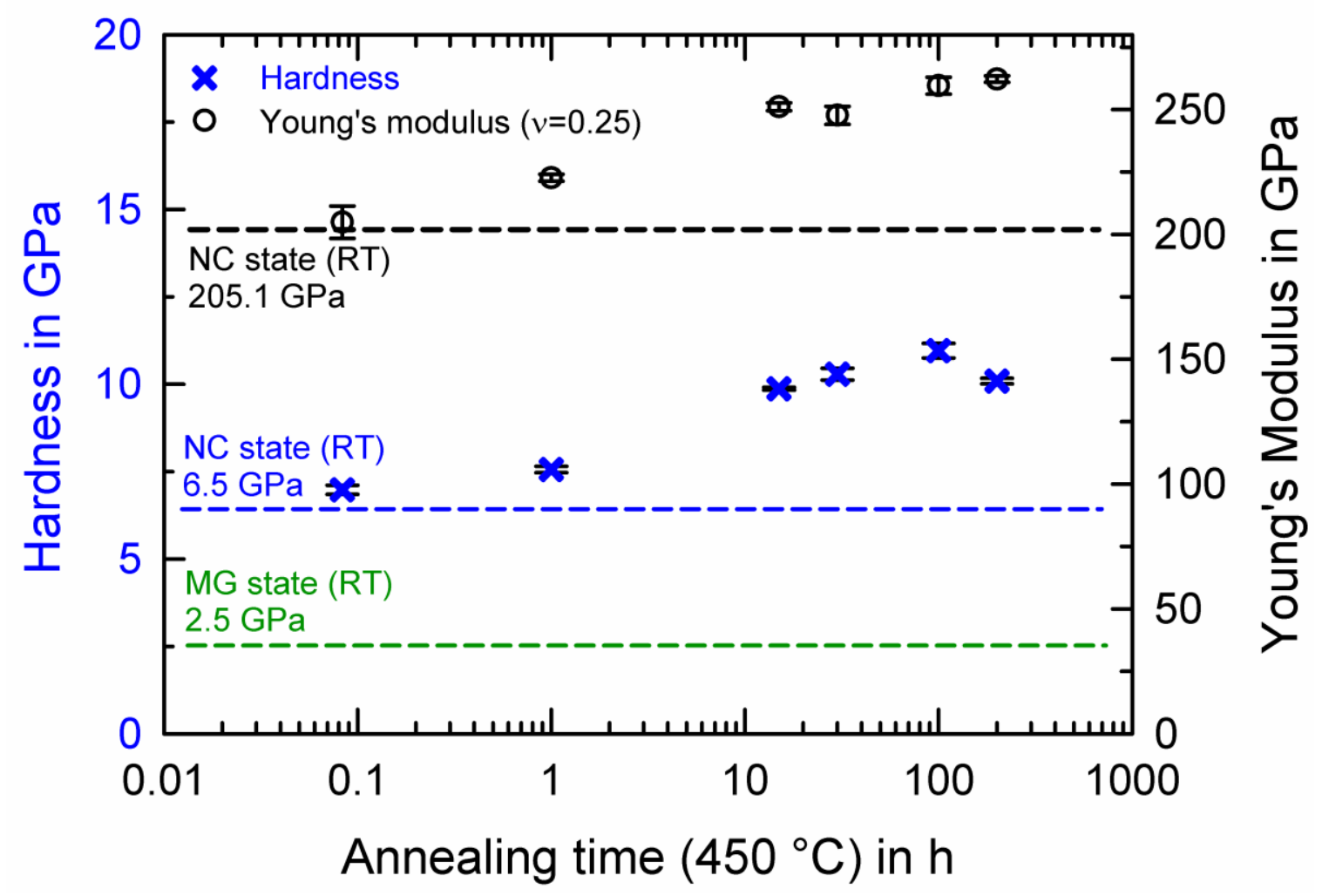


Figure 5. Room-temperature nanoindentation strain rate jump tests on the different material states. a) Load-displacement curves with inset showing Young's modulus vs. indentation depth. b) Hardness vs. indentation depth. c) Magnified view of hardness vs. indentation depth for strain rate jump at $1500 \mathrm{~nm}$.

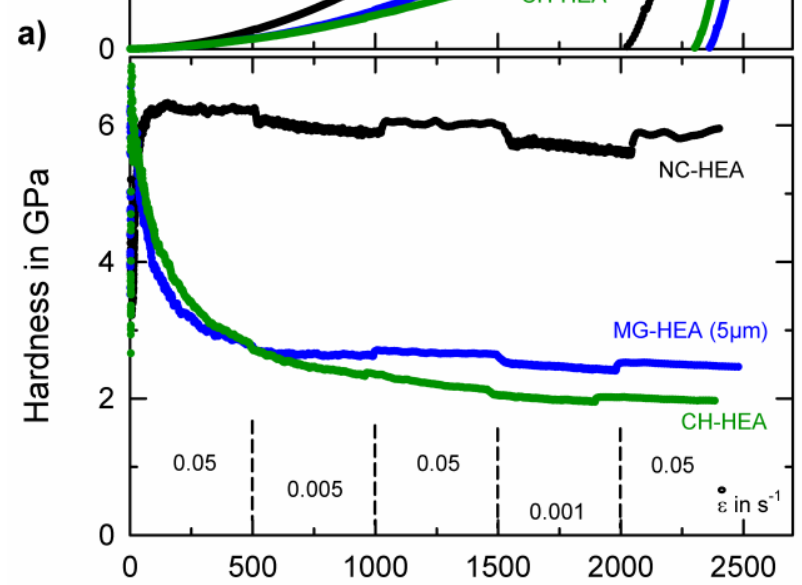

b) Indentation depth in $\mathrm{nm}$

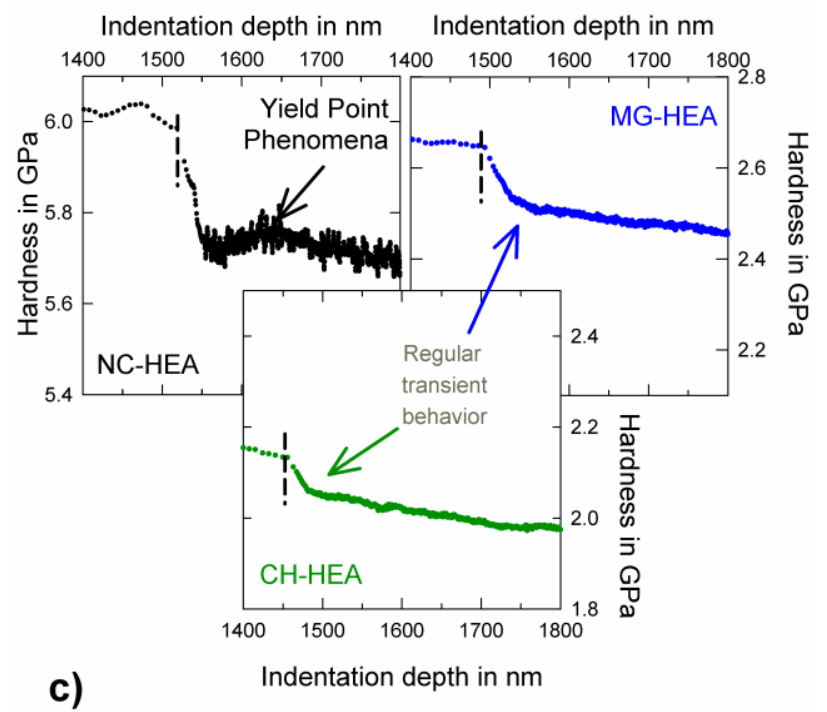


Table 1.

Strain rate sensitivity $m$ and activation volume $V^{*}(b \sim 2.53 \AA)$.

\begin{tabular}{c|cc}
\hline Microstructure & $m(-)$ & $V^{*}(b 3)$ \\
\hline CH state $(d>1 \mathrm{~mm})$ & 0.008 & 69 \\
MG state $(\mathrm{d} \sim 6 \mu \mathrm{m})$ & 0.014 & 42 \\
NC state $(\mathrm{d} \sim 501 \mathrm{~nm})$ & 0.018 & 16
\end{tabular}




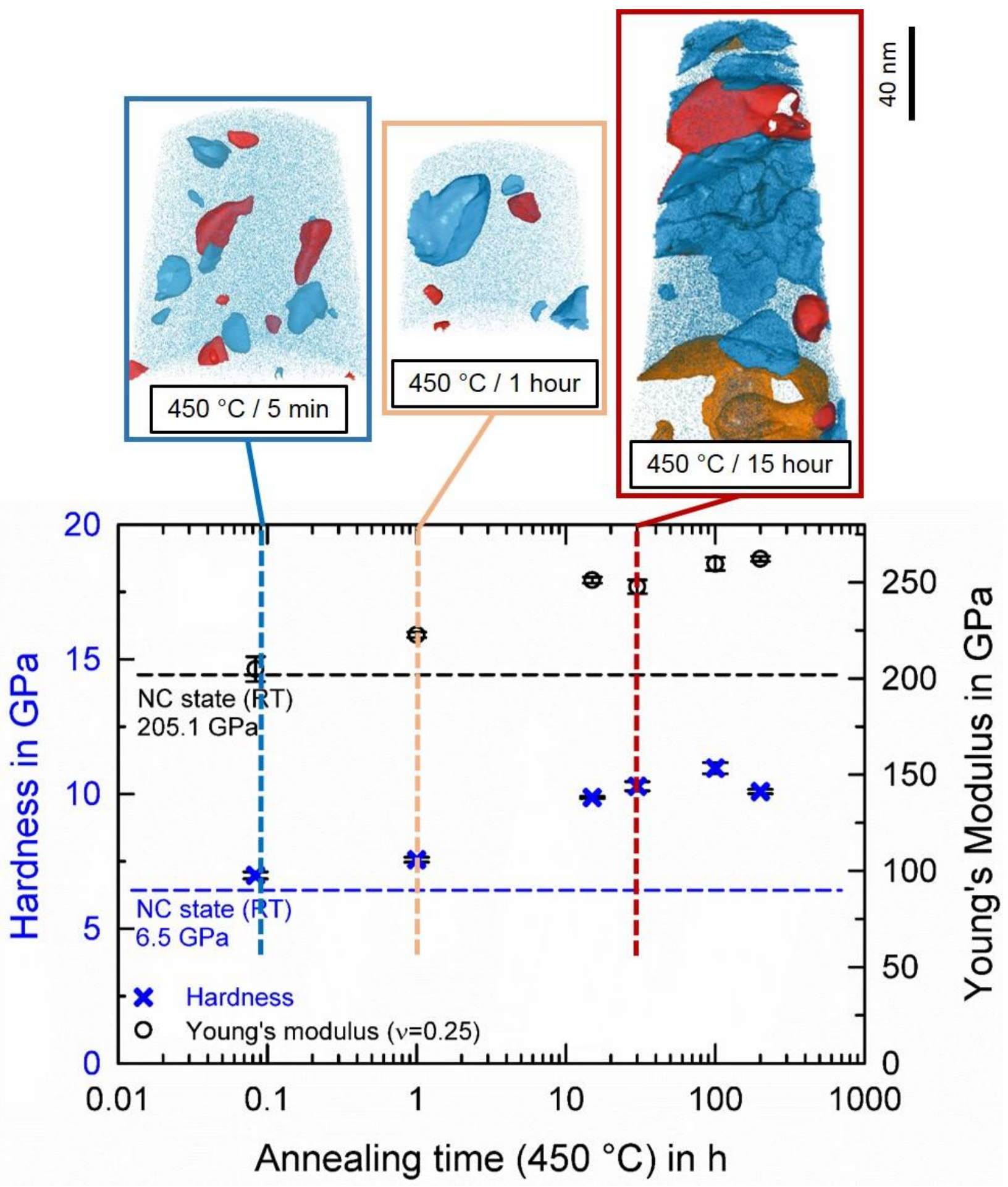

